
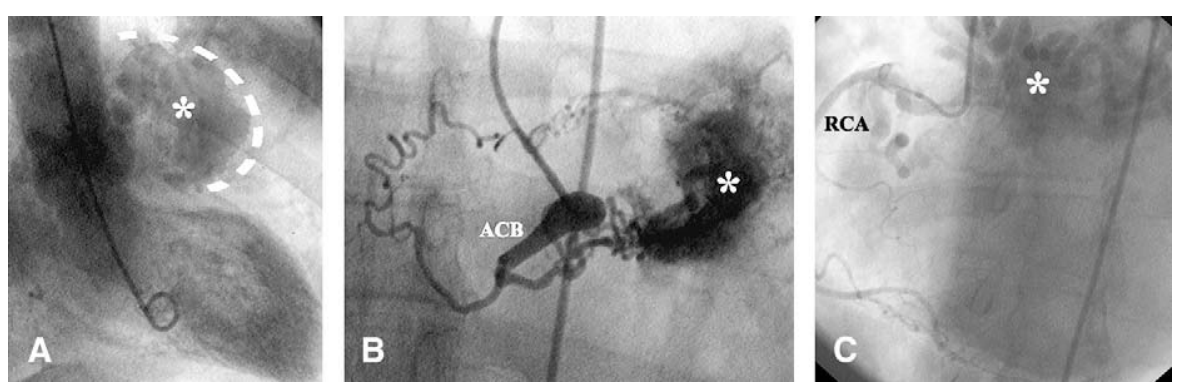

FIGURE 1. Preoperative coronary and aortic angiogram. A, Left ventriculography and related aortic angiographic analysis showing the location of the primary paraganglioma and the anatomic relationship with the cardiovascular structures. B, Perfusion of the primary paraganglioma from a branch of the circumflex coronary artery. C, A second perfusion branch of the primary paraganglioma arises from the proximal portion of the right coronary artery, showing a tortuous course and initial signs of vessel aneurysm.
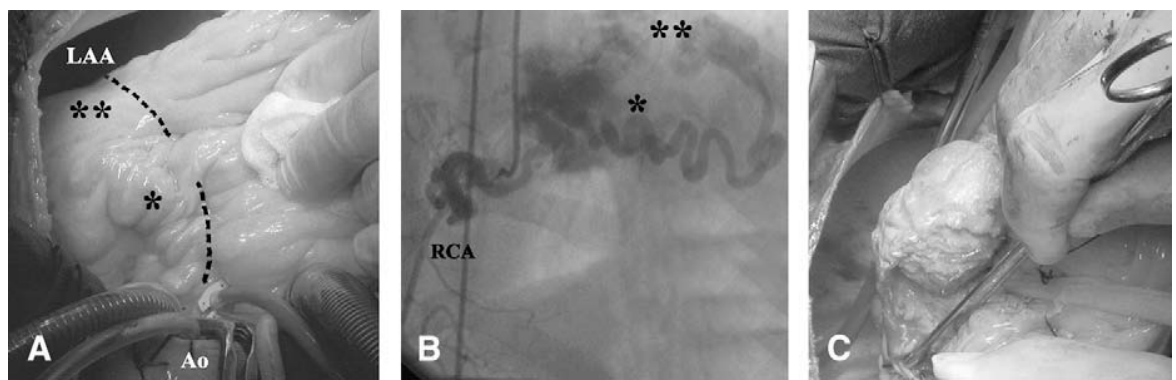

FIGURE 2. Intraoperative view of the tumor. Panel A shows the ventricular vision after sternotomy and pericardiotomy: the ventricular mass is evident (double asterisk), showing a coronary branch (asterisk) coming from the right coronary artery. Panel B shows the entire course of the main feeding branch of the primary paraganglioma, coming from the right coronary artery and ending in a sort of vascular fistula. $\mathrm{C}$, The tumor excision is documented, showing the intimate relationship and depth at the left ventricular level.

unresectable. The use of chemotherapy and radiotherapy, in such cases, is of limited value. ${ }^{5}$

\section{References}

1. Lee HH, Brenner WI, Vardhan I, Hyatt J, Terlecki M. Cardiac pheochromocytoma originating in the interatrial septum. Chest. 1990;97:760-2.
2. Orringer MB, Sisson JC, Glazer G, et al. Surgical treatment of cardiac pheochromocytomas. J Thorac Cardiovasc Surg. 1985;89:753-7.

3. Chan JKM, Pontefract D, Andrews R, Naik SK. Paraganglioma of the left atrium. J Thorac Cardiovasc Surg. 2001;122:1032-3.

4. Jebara VA, Uva MS, Farge A, et al. Cardiac pheochromocytomas. Ann Thorac Surg. 1992;53:356-61.

5. Vander Salm TJ. Unusual primary tumors of the heart. Semin Thorac Cardiovasc Surg. 2000;12:89-100.

\title{
A case of antiphospholipid syndrome presenting with a floating thrombus in the ascending aorta
}

Yang Gi Ryu, MD, ${ }^{\text {a }}$ Cheol Hyun Chung, MD, ${ }^{a}$ Suk Jung Choo, MD, ${ }^{a}$ Yun Seok Kim, MD, and Jae Kwan Song, MD,

Seoul, South Korea

From the Departments of Thoracic and Cardiovascular Surgery ${ }^{a}$ and Internal Medicine, ${ }^{\mathrm{b}}$ ASAN Medical Center, University of Ulsan, College of Medicine, Seoul, South Korea.

Received for publication Jan 23, 2008; accepted for publication Jan 26, 2008.

Address for reprints: Cheol Hyun Chung, MD, Department of Thoracic and Cardiovascular Surgery, ASAN Medical Center, 388-1 Poongnap-dong, Songpa-gu, Seoul, Republic of Korea, 138-736 (E-mail: hyun227@amc.seoul.kr).

J Thorac Cardiovasc Surg 2009;137:500-2

$0022-5223 / \$ 36.00$

Copyright (c) 2009 by The American Association for Thoracic Surgery

doi:10.1016/j.jtcvs.2008.01.028
We report the case of a patient with primary antiphospholipid syndrome (APS) with a presenting symptom of dyspnea. Transesophageal echocardiography and aortic dissection computed tomography showed a floating thrombus in the distal ascending aorta.

\section{CLINICAL SUMMARY}

A 57-year-old man was admitted with the symptom of gradual dyspnea for 1 month. He had no medical history of 

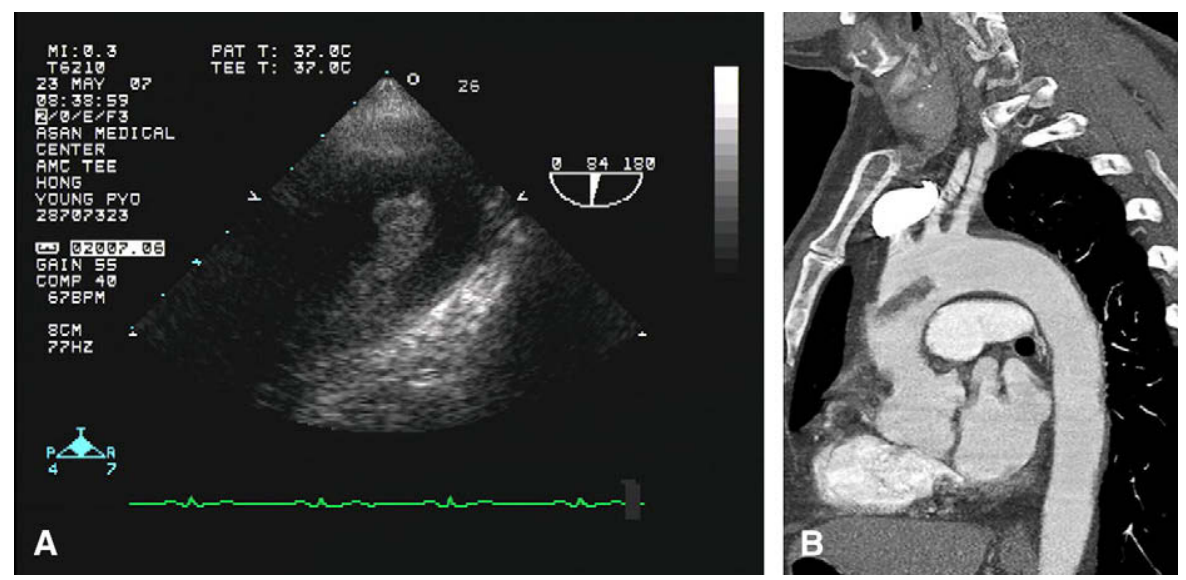

FIGURE 1. Preoperative evaluation. A, Transesophageal echocardiographic image of intra-aortic floating thrombus. B, Computed tomographic image of intra-aortic floating thrombus.

arrhythmia, ischemic heart disease, diabetes mellitus, or stroke. Results of his general physical examination were also unremarkable. Routine laboratory values were as follows: hemoglobin, $14.3 \mathrm{~g} / \mathrm{dL}$; white blood cell count, 5.7 $\times 10^{3}$ cells $/ \mathrm{mm}^{3}$; platelet count, $212 \times 10^{3} / \mathrm{mm}^{3}$; and prothrombin time and activated partial thromboplastin time both within their reference ranges. Erythrocyte sedimentation rate, C-reactive protein level, renal and liver function values, and urinalysis results were also within reference ranges. Results of the thrombophilia screen also proved normal, except for positive IgG, IgM anticardiolipin, and IgM anti- $\beta_{2}$-glycoprotein I antibodies; that is, IgG phospholipids at $21 \mathrm{U} / \mathrm{mL}$ (reference $<10 \mathrm{U} / \mathrm{mL}$ ), IgM phospholipids at 22 $\mathrm{U} / \mathrm{mL}$ (reference $<10 \mathrm{U} / \mathrm{mL}$ ), and IgM phospholipids at 32 $\mathrm{U} / \mathrm{mL}$ (reference $<20 \mathrm{U} / \mathrm{mL}$ ), respectively. Transesophageal echocardiography showed a large, free-floating thrombus in the distal ascending aorta (Figure 1, A). It appeared to be attached just proximal to the right innominate artery, with extension into the aortic arch. After initial intravenous anticoagulation with heparin, reassessment 3 days later with aortic dissection computed tomography (Figure 1,B) showed no significant change. Because of the risk of further distal embolism, the decision was made to urgently remove the thrombus surgically. The aorta was exposed through a median sternotomy. Right axillary arterial and selective bicaval venous cannulations were performed, followed by core cooling to $30^{\circ} \mathrm{C}$. Cardiac arrest was induced with retrograde cardioplegic solution through the coronary sinus. Total circulatory arrest was then instituted, and continuous antegrade cerebral perfusion was established through right axillary perfusion after crossclamping of the right innominate and left common carotid arteries for 16 minutes. The aortic lumen was exposed through a longitudinal incision extending from the mid ascending aorta to just below the right innominate artery without distal crossclamping (Figure 2, A). A thrombus longer than $5 \mathrm{~cm}$ (Figure 2, B) was removed from the proximal aortic arch. A smaller amount of residual thrombus was removed from the roughened surface of the aorta. The intima at the base of the thrombus appeared relatively normal after thrombus removal. The aorta was repaired with a 4-0 polypropylene running suture. The left common carotid and right innominate arteries were then unclamped, and whole-body perfusion was reestablished. Histopathologic examination revealed the mass to be an organized thrombus without evidence of malignancy. The patient was discharged on postoperative day 10 with a regimen of warfarin sodium after an uneventful recovery. The patient continues to do well at 6 postoperative months of follow-up.
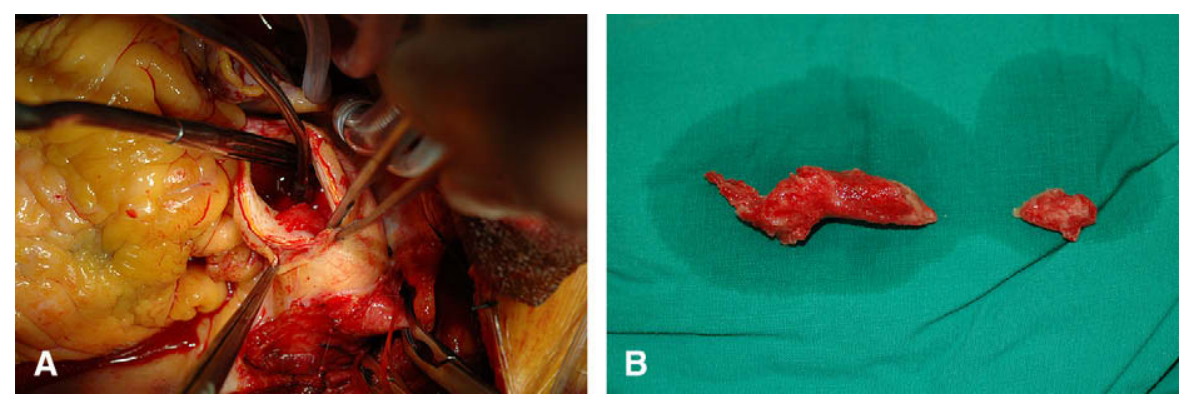

FIGURE 2. Intraoperative findings. A, Aortic lumen exposed through longitudinal incision. B, Organized thrombus. 


\section{DISCUSSION}

APS is defined by the occurrence of arterial or venous thromboses in the presence of antiphospholipid antibodies, namely anticardiolipin antibodies or lupus anticoagulant. ${ }^{1}$ The cardiovascular system is a commonly affected target for APS-associated morbidity, resulting in endocardial disease, intracardiac thrombosis, and various myocardial lesions. The successful removal of a large, spontaneously developed aortic thrombus is described in this case. APSrelated cardiac disorders were initially described by Kaplan and colleagues ${ }^{2}$ in 1992, and are currently seen in as many as $76 \%$ of patients with APS. ${ }^{2-3}$ Because floating thrombi in the ascending aorta may lead to life-threatening complications, rapid and reliable methods of diagnosis and treatment are essential. Transesophageal echocardiography has conventionally been used for the evaluation of intracardiac masses and the aorta. Thrombolysis and anticoagulation have been reported in the literature, ${ }^{4}$ but they carry the risk distal embolism from partial lysis and dislodgment of the thrombus. This line of management was therefore abandoned, and an operation was recommended as the treatment of choice. Sadony and coworkers ${ }^{5}$ have described successful removal of thrombus with hypothermic circulatory arrest. In our case, it was possible to remove the distal ascending aorta thrombus without hypothermic circulatory arrest by instituting cardiopulmonary bypass through bi- caval venous and axillary arterial cannulations. The increased risk of coronary or cerebral embolization from the floating thrombus was a concern with femoral arterial cannulation. Our technique was simpler, faster, and safer, because we did not need to lower the systemic body temperature as much and also were able to inspect the aortic lumen fully from the aortic root to the proximal descending aorta.

This case demonstrates the feasibility of safe and rapid extraction of aortic intraluminal thrombus by means of selective bicaval venous drainage and axillary arterial perfusion. This approach obviates the increased risk of comorbidities associated with prolonged cardiopulmonary bypass required for deep hypothermia and the potential risk of coronary or cerebral embolism from retrograde arterial perfusion.

\section{References}

1. Hughes GR, Harris EN, Gharavi AE. The anticardiolipin syndrome. J Rheumatol. 1986;13:486-9.

2. Kaplan SD, Chartash EK, Pizzarello RA, Furie RA. Cardiac manifestations of the antiphospholipid syndrome. Am Heart J. 1992;124:1331-8.

3. Diana B, David B, Roderic T. Left ventricular thrombi in a patient with the antiphospholipid syndrome. Br Heart J. 1995;74:202-3.

4. Hausman D, Gulba D, Borgheer K, Niedermeyer J, Comecs KA, Daniel WG. Successful thrombolysis of an arch thrombus in a patient after mesenteric embolism [letter]. N Engl J Med. 1992;327:500-1.

5. Sadony V, Walz M, Lohr E, Rimpel J, Richter HJ. Unusual cause of recurrent arterial embolism floating thrombus in the aortic arch surgically removed under hypothermic cardiocirculatory arrest. Eur J Cardiothorac Surg. 1988;2:469-71. 